XIV.

Aus der Berliner städtischen Anstalt für Epileptische Wuhlgarten.

\title{
Haemangiom der weichen Hirnhaut bei Naevus vasculosus des Gesichts ${ }^{1}$ ).
}

Von

Direktor Dr. Otto Hebold.

Bei dem kleinen, jetzt nahezu 13 Jabre alten Knaben, den ich hier vorstelle, ist, wie Sie sehen, die linke Kopfhälfte von einem ausgedehnten Naevus vasculosus eingenommen. Er betrifft die linke Gesichtshälfte, nur die Gegend des Unterkiefers und ein schmaler Streifen vor dem $0 \mathrm{hr}$ ist frei davon. Der Naevus geht in Streifen auf die rechte Gesichtshälfte hinüber. Es finden sich Streifen an der Stirn und am rechten oberen Augenlid. Die Bindehäute beider Augen sind ebenfalls rot. Die Mundschleimhaut ist in der linken Hälfte gerötet und auch etwas gewulstet, wie die ganze linke Hälfte der Oberlippe. Die linke Gesichtshälfte ist stärker entwickelt. Der Schädel ist im rechten schrägen. Durchmesser verkürzt. Kopfmasse 15:16:50 cm. Die Pupillen sind gleich und mittelweit, der linke Optikus ist hyperämisch, unscharf begrenzt, die peripapilläre Zone von einem Exsudat eingenommen; zwei kleine Arterien (temporal unten) sind von Exsudat vollständig bedeckt (Neuroretinitis). Die Gesichts felder sind nicht auffallend eingeschränkt. Die Zunge wird nur mühsam zitternd vorgestreckt, weicht dabei deutlich nach links ab. Der Gaumen wird beiderseits gut gehoben. Der rechte Arm ist kürzer und schlechter entwickelt, die Spannung ist in der rechten Schulter und im rechten Ellenbogen gegen links erhölnt. Bewegungen der Schulter geschehen ausgiebig, aber schwächer wie links. Der Händedruck ist rechts schwächer, links sehr kräftig. Die rechte Hand ist kleiner wie die linke: die Reflexe sind rechts lebhafter. Die Beine scheinen nahezu gleich entwickelt, jedoch ist die rohe Kraft rechts herabgesetzt, auch: ist das rechte Bein kürzer, der Gang schleppend. Kein Fussklonus, Babinski rechts vorhanden, Oppenheim rechts angedeutet. Stiche werden beiderseits. lebhaft empfunden. Der Penis ist auffallend klein, die Hoden sind richt im Hodensack. Das Fettpolster ist stark entwickelt.

1) Vortrag im Psychiatrischen Verein zu Berlin am 14. 12. 12. 
Die Sprache ist kindlich, Doppelkonsonanten machen ihm Mühe, 1 nach b ist ihm unmöglich, statt Blei sagt er z. B. "Brei", statt Schrank ..Schank“Platte "Patte".

Er schreibt nur seinen Namen, und zwar mit der linken Hand. Der stereognostische Sinn ist links gut, rechts sehlecht. Er zeigt aber mit der rechten. Hand richtig nach Ohr und Nasenspitze. Geruch beiderseits gut, Geschmack auch. Das Gefühl ist in der rechten Rumpfseite und im rechten Arm herabgesetzt, das Schmerzgefühl gesteigert. Der Kranke ist geistig sehr zurückgeblieben. Er jst viel für sich allein, immer freundlich, liest viel. Er hatte hier wiederholt eigenartige Anfälle, die übrigens nicht häufig sind. Ein Anfall wird folgendermassen beschrieben: Pat. stand beim Baden unter der Brause, lief plötzlich vom Wasser weg, drehte sich im Kreise rechts nach hinten um und brachte dabei unverständliche, singende Töne hervor, die Augen waren nach oben rechts gedreht, die Gesichtsfarbe nicht verändert, die Pupillen weit, liessen sich bei der Unruhe des Kranken nicht prüfen. Auf Nadelstiche zuckte er. Der Zastand dauerte etwa 5 Minuten.

Die ersten epileptischen Anfälle hatte er vom 4. bis 9. Monat ohne äussere Veranlassung. Damals hatte er viel mit den Zähnen zu tun. Er hatte 18 bis 19 Anfälle am Tage. Sie haben bis zum 7. Jahre ausgesetzt. Dann kamen sie wieder in Zwischenräumen von 2 bis 3 Tochen, gehäuft mehrere Tage hintereinander. Er bokam sogar Anfälle, wenn er sich freudig erregte. Die Zuclíngen betrafen immer nur die rechte Seite; dabei fängt es am Fuss an, er fällt um, hat im Arm keine Zuckungen, der Mund wird nach rechts verzogen, der Blick wird starr, dann läuft er herum, fasst alles an, kann nicht sprechen. 10 Minuten später kann er wieder lesen. Er nässt dabei ein, beisst sich nicht auf die Zunge. Die Anfälle verschlimmerten sich, es trat sogar Erbrechen auf, oft schreit or laut. In der Schule kam er nur zur 3. Nebenklasse.

Ein Onkel der Mutter war Idiot. Eine Schwester der Mutter des Vaters hatte Krämpfe. Er ist ein Arbeitersohn, beide Eltern sind gesund.

Ich reihe dem einen zweiten Fall an, der nicht so hochgradige Störungen zeigt.

Die Arbeiterin Cl. E., geb. den 19. März 1893, ist insofern crblich belastet, als der Vater trinkt, die Mutter des Vaters geisteskrank gewesen ist. Sie war rom Kassenarzt in die Anstalt geschickt, weil sie die rechte Hand nicht richtig bewegen könne. Sie habe oft, gab sie an, solch ein Zittern in der rechten Hand bekommen, beim Arbeiten sei ihr einmal am 26. 8. 11, 3 Wochen etwa vor ihrer Aufnahme in der Anstalt, der Schraubenzieher aus der Hand gefallen. Krampf- oder Schwindelanfälle habe sie niemals gehabt. Alle Gèschwister sind gesund. Auch sie war bisher gesund, auch nicht geschlechtskrank, hatte mit 12 Jahren ihre erste Regel, seit 2 Jahren Geschlechtsverkehr, sei jetzt schwanger seit Mai. Sie ist aber in der Schule nur bis zur 3. Klasse gekommen, besonders das Rechnen fiel ihr schwer, faul sei sie nicht gewesen. Thre Schulkenntnisse sind mangelhaft. Sie ist seit einem Monat ron Hause fortgegangen, weil sie der Mutter nicht gehorchen und viel ausgehen wollte. Seitdem ging sie in eine Fabrik. 
Sie ist von kleiner Gestalt, gutem Ernährungs- und Kräftezustand. Der Schädel ohne Besonderheit. Auf der Stirn über dem rechten Auge befindet sich ein kleinhandtellergrosser Naevus vasculosus. Motilitäts- und Sensibilitätsstörungen sind nicht vorhanden. Die linke Pupille ist weiter, die rechte mittelweit, die Lichtreaktion links träge, rechts lebhaft, Konvergenzreaktion gut. Die Patellar-, Achillessehnen- und Fussreflexe sind gleich, von mittlerer Stärke. Kein Babinski. Die Bewegungen der rechten Hand haben etwas Choreatisches.

In diesem Falle ist ebenfalls geistige Schwäche vorhanden und hat sich ein Reizzustand in der motorischen Gegend des Armes, aber der dem Naevus entgegengesetzten Gehirnseite entwickelt. Aber dies ist nicht der einzige Unterschied dem rorgestellten Falle gegenüber. Es sind auch alle Erscheinungen sehr gering.

Bei dem Knaben liegt ein deutlicher Fall von zerebraler Kinderlähmung vor, wobei die Gehirnschädigung die Seite betrifft, die äusserlich durch einen Naevus vasculosus von grosser Ausdehnung gezeichnet ist. Die hochgradige geistige Schwäche, die halbseitigen Erscheinungen seitens des Nervensystems, die epileptischen Anfälle deuten darauf hin, dass die linke Grosshimhälfte hochgradig beeinträchtigt sein muss, die Hirnhälfte der Seite, die äusserlich von dem grossen Gefässmal eingenommen ist.

Dass hier kein zufälliges Zusammentreffen vorliegt, beweisen mir andere Fälle, die zur Obduktion gekommen sind. Dafür spricht auch die Beobachtung anderer, wenn auch durch sie nicht der ursächliche Zusammenhang ganz aufgeklärt worden ist. Ich verweise zunächst auf Lannois und Bernoud. (Enorme naevus-angiomateux de la face avec hemiplégie spasmodique et épilepsie. Nouv. Iconogr. de la Salpêtrière. T. XI. p. 446.) Diese betonen das Zusammentreffen solcher Angiome mit den Bildungsfehlern, die zur Ursache der Idiotie, Epilepsie usw. werden können. In dem einen Fall fand sich linksseitige Parese bei linksseitigem Nävus. In einem zweiten Falle war rechtsseitige Parese bei linksseitigem Angiom vorhanden. Kalischer (Dieses Archiv, Bd. XXXIV, S. 171) teilt einen „Fall von linksseitiger Teleangiektasie (Angiom) des Gesichts und der weichen Hirnhaut" mit schöner Abbildung mit und geht auch auf frühere Mitteilungen ein. Solche sind auch in Oppenheims Lehrbuch der Nervenkrankheiten, 1908, Bd. II, S. 1014, erwähnt. Naecke (Ein Beitrag zur Pathogenese des Naevus vascularis, Neurol. Zentralbl., 1905, Bd. XXIV. S. 930) bespricht einen Fall und sucht nach einer Erklärung des Zusammenhanges dieses Stigmas, wie er es nennt. Ihm scheint für die meisten Teleangiektasien und Naevi der teratologische bzw. irritative Ursprung schwach angelegter Gefässe, soweit nicht blosse Stautung in Frage Kommt, die einzig richtige Erklärung zu sein. In seinem Beispiel handelt es sich 
ebenfalls um einen von Geburt schwachsinnigen Menschen mit linksseitigem Naevus. Ein weiterer Versuch der Erklärung der Naevi ist der von Cushing (Of spontaneous internal haemorrhage associated with trigeminal nevi. The journ. of the Amer. med. ass. 21. Juli 1906. p. 178.). Er sagt, sie entsprechen in ihrer Ausbreitung gewöhnlich einem oder mehreren Trigeminusästen. Sie seien mit einer Hypertrophie der tieferen Gewebsschichten des Gesichts usw. oder mit ähnlichen Veränderungen der Dura mater verbunden. Dieser durale Naevus könne zu spontaner Blutung in der Kindheit mit entsprechenden Folgen führen, wie Epilepsie, spastischer Hemiplegie und selbst Demenz. Diese Deutung der Entstehung und der zerebralen Befunde stimmt indessen nicht. Es handelt sich nicht um Hämorrhagien innerhalb des Schädels. Die Erklärung geben neben den Beobachtungen anderer drei meiner Fälle, die zur Obduktion kamen, nachdem ich sie längere Zeit zu beobachten Gelegenheit gehabt hatte.

1. Fall. A. H., beim Tode 32 Jahre alt, hatte einen rechtsseitigen grossen Naevus vasculosus im Gesicht und linksseitige Parese mit Kontrakturen und Hypoplasie der Gliedmaassen. Es fand sich rechtsseitige Hypoplasie des Grosshirns mit Mikrogyrie und linksseitige des Kleinhirns. Während die Pia der linken Seite zart war, war die der rechten verdickt, die Gefässe dieser Seite hatten dicke Wandungen, waren geschlängelt und die Pia sass an den hinteren Absehnitten fest. An der Spitze des Hinterhauptlappens war eino Stelle mit. Kalk durchsetzt. Die harte Hirnhaut war rechts mit einem Pigmenthäutchen bedeckt.

2. Fall. 0. S., geb. 15. 11. 81, gest. 4. 9. 04, hatte einen grossen Naevus vasculosus, der die linke Kopfhälfte bis auf den Hals einnahm. Er war rechtsseitig paretisch, zeitweilig völlig rechtsseitig gelähmt und hatte sehr häufig epileptische Anfälle eigener Art, die als Rucke bezeichnet wurden, aber auch solche mit Zuckungen. Er fiel ohne Vorboton hin, meist auf das Gesicht, so dass die Lippe jedesmal stark anschwoll, und erhielt dagegen eine Schutzkappe. Dazu bestand hochgradige geistige Schwäche. Hier ergab die Obduktion ein linksseitiges Hämangiom der $\mathrm{Pia}$ und Hypoplasie der ganzen linken Grosshirnbälfte.

Der dritte Fall betrifft eine Kaufmannstochter. F. H., geb. 29. 3. 71 gest. 18. 2. 99. Hier nahm der grosse Naevus fast das ganze Gesicht, die Rückseite des Thorax und die rechte Vorderseite der Brust ein. Nach einer angeblichen Gehirnhautentzündung im 3. Lebensjahre hatten sich Krämpfe eingestellt und war sie vollkommen verblödet. Die Krämpfe waren häufig, bald rechtsseitig, bald allgemeiner. Dazu bestand Lähmung sämtlicher Gliedmassen mit Kontrakturen, Blasen- und Mastdarmlähmung. Aus dem Obduktionsbefund ist hervorzuheben: Die rechte Hälfte des Stirnbeins ist doppelt so dick wie die linke, die Dura ist am rechten Stirnteil verdickt, die Pia an der mittleren Kante auffallend verdickt und getrübt, der Plexus chorioideus an einer Stelle 
ganz hart, bindegewebig entartet. Dann fand sich Hypoplasie der rechten Kleinhirnhälfte und der rechten Grosshirnhälfte, dazu war der rechte Schläfenlappen und Hinterhauptslappen an der Aussenseite erweicht und mit Kalkkörnchen durchsetzt. Letztere finden sich auch an der Spitze des Stirnlappens.

Wenn wir diese Befunde auf den vorgestellten Fall anwenden, so werden wir bei der Gleichartigkeit aller Erscheinungen nicht fehl gehen, wenn wir auch hier annehmen, dass sich in Innern des Schädels ein ähnlicher Vorgang wie in den Bedeckungen des Schädels und im Gesicht abspielt und dass auch hier die Ausbreitung des krankhaften Vorganges eine so ausgedehnte ist, wie in meinen beiden erstgenannten Fällen, die zur Obduktion kamen.

Es kann angezweifelt werden, ob der dritte Fall hierher gehört. Doch habe ich ihn nicht nur der Vollständigkeit halber erwähnt; ich glaube, er kann auch hier eingereiht werden. Ersichtlich ist der krankhafte Vorgang im Gehirn wesentlich rechts verlaufen und der Naevus hatte ebenfalls rechts seine grösste Ausdehnung. Die Lähmungserscheinungen waren zwar doppelseitige, aber es muss bei dem hochgradigen Blödsinn der Kranken dahingestellt bleiben, wie weit Kontrakturen durch Inaktivität herbeigeführt waren, und die Lähmung der linken Seite eine Folgeerscheinung war. Und was nun das Fehlen der Gefässvermehrung (des Hämangioms) der Gehimhäute angeht, so möchte ich darauf hinweisen, dass hier sicher ein Rückbildungsvorgang vorliegt, der einzelne Rückstände in der auffallend verdickten Pia und in der bindegewebigen Entartung des Plexus der rechten Seite zurückgelassen hat, während die Gehirnverringerung beim Abschluss der Entwicklung bestehen bleiben musste. Ich mache hier auch auf den gleichartigen, aber hochgradigeren Befund von Kalkansammlung in diesem und in dem ersten Falle aufmerksam. Die Annahme der Rückbildung der Gefässgeschwulst in der Pia stützt sich auf die Beobachtung der Chirurgen bei den Hauthämangiomen. So sagt Bergmann bei der Behandlung des Gegenstandes im. praktischen Handbuch der Chirurgie von Bergmann, Bruns und Mikulicz, Bd. I, S. 33, dass die grossen, gleichmässig blassrot gefärbten, flächenhaften Teleangiektasien ohne jede Niveaudifferenz mit der übrigen Haut sich in der Regel von selbst zurückbilden. Nicht selten geschehe die Rückbildung auf dem Wege der Entzündung und Ulzeration mit nachfolgender Vernarbung und Schrumpfung der ganzen Geschwulst. Und bei diesem Gehirn sind gerade die Folgeerscheinungen der Entzündung auffallend.

Das gleichzeitige Vorkommen dieser Bildung in den weichen Bedeckungen und im Innern des Schädels - auch verbunden mit Verdickungen des Schädels an derselben Seite — weist darauf hin, dasis 
hier ein gleichzeitig ausgelöster kranklafter Vorgang rorliegen muss. Es ist bekannt, dass diese Naevi, wenn sie auch nicht gleich voll entwickelt sind, angeborene Gebilde sind. Der Lieblingssitz ist der Kopf mit den anstossenden Teilen des Halses und Nackens. Nun hat Virchow (Die krankhaften Geschwülste, Bd. III, S. 345 u. 406) die Erklärung gegeben, dass alle jene Punkte des Kopfes besonders ausgesetzt sind, wo in embryonaler Zeit Spalten bestehen, die sich in einer späteren Zeit des Intrauterinlebens schliessen. „Es kommen hier namentlich in Betracht die Kiemenspalten, zumal die oberen, sowie die Mund-Nasenspalte und die davon abgehende Tränenspalte, welche zur Augenspalte führt. Die obere Kiemenspalte reicht bekanntlich ursprünglich bis in das Ohr hinein, die Mund-Nasenfalte reicht einerseits bis zur Stirn, andererseits bis tief in die Wangengegend. Mancherlei Missbildungen schliessen sich an diese embryonalen Verhältnisse an ... wir wissen, dass die Entwicklung der Gefässe sich vielfach an die Geschichte jener Spalten anschliesst. Sehr leichte irritative Zustände mögen genügen, um an den Rändern und im Umfange dieser Spalten, welche an sich sehr reich mit Gefässen versehen sind, eine stärkere Ausbildung derselben hervorzurufen, die sich möglicherweise als Naevus zu erkennen gibt, die aber auch wohl ganz latent bleibt und erst später manifest wird".

Ich glaube, alle diese Ausführungen werden durch unsere Beobachtung gestützt. Das gleichzeitige Vorkommen des Naevus in den Bedeckungen des Schädels und in der weichen Hirnhaut erklärt sich aus derselben ursprünglichen Anlage dieser Gebijde und muss daher eine frühzeitige Missbildung sein. Denn sowohl die äusseren Bedeckungen des Schädels wie alle Hirnhäute gehen aus Schichten des Primordialkraniums hervor, aus dem sonst nur noch ein Teil des Schädels sich entwickelt (Kölliker, Entwicklungsgeschichte des Menschen und der höheren Tiere. Leipzig 1879. S. 433.) Dieses häutige Kranium hat, abgesehen von den Durchtrittsstellen der Nerven und Gefässe, nur vorübergehend an einer Stelle eine Unterbrechung in der Gegend der späteren Sella turcica und schliesst sich auch bald wieder. Es muss daher, da die gleiche Veränderung sich in den aus dem Primordialkranium innerhalb und ausserhalb des Schädels entstehenden Gebilden zeigt, eine sehr frühzeitige Störung angenommen werden, wo die Gefässe der embryonalen Anlage, die diese Gebilde versorgen, noch eine gemeinschaftliche Gefässlücke haben oder in Zusammenhang sind. Es liegt nahe, anzunehmen, dass die Rückbildung der ursprünglichen Gefässverzweigungen gestört wird, ursprüngliche Gefässe bleiben und bei dem dem embryonalen Gewebe besonders innewohnenden Wachstumstriebe neue Netze sich bilden und dass dies sich nach der Geburt noch fortsetzt. Es 
kann aber auch später noch eine Rückbildung eintreten, wie es oben erwähnt und für den letzten Fall, was die Gefässgeschwulst in der Pia angeht, als wahrscheinlich angenommen worden ist. Vielleicht führt der Umstand, dass die lissbildung in den meisten Fällen eine linksseitige ist - denn das ist wohl kein Zufall -, die Entstehung derselben einer Erklärung näher. Es kommen hier zweifelsohne Vorgänge in Betracht, die mit der endgültigen Entwicklung des Gefässsystems an Kopf und Gehirn in Zusammenhang stehen. Es ist ja bekannt, wie gerade die Verhältnisse am Gefässsystem mancherlei Wandlung durchmachen; wie ursprünglich gefässreiche Gegenden eingehen oder zurückgehen und die Gefässverzweigung sich ganz bedeutend ändert. Dabei hat jede Körperhälfte ihre Eigenheiten in der Gefässentwicklung, in der Abzweigung der grossen Stämme und in der Gefäss- und Blutverteilung. Hier stehen die arteriellen Kiemenbögen voran. Vielfältiger als bei den Arterien ist die Entwicklung bei den Venen. Während die Gefässe ursprünglich paarig geordnet sind, macht in der weiteren Entwicklung. die symmetrische Anordnung der Jugular- und Kardinalvenen einer asymmetrischen Platz, unter Rüclzbildung und Verkümmerung einiger Hauptstämme, besonders der linken Seite.

Die Gefässmissbildung in der Pia ruft nun eine Störung in der Entwicklung des Gehirns hervor, indem die davon betroffene Grosshirnhälfte sich zwar eine Zeitlang in allen Teilen gleichmässig bis zu einer gewissen Grösse entwickelt, dann aber im Wachstum stehen bleibt und bei weiterem Bestehen Rückbildungen eintreteu. In dem dritten Falle fand sich auch eine Hypoplasie des Kleinhirns, aber derselben Seite; man wird annehmen müssen, dass hier die Störung anch an dem Kleinhirn selbst angegriffen hat, sonst würde man, wenn überhaupt eine, dann die Hypoplasie der anderen Kleinhirnhälfte zu erwarten gehabt haben, wie im Fall 1. Dass in dem zweiten Falle das Kleinhirn unbeteiligt ist, beruht wohl darauf, dass der krankhafte Vorgang in der Gefässhaut nur eine langsame Wirkung ausübt. Hierauf weist ja auch die gleichmässige Entwicklung der betroffenen Gehirnhälfte hin. Ist sie bis zu einer gewissen Grösse gediehen, dann tritt der Zeitpunkt ein, wo die Ernährung unzureichend wird, sei es, dass es schon von vornherein in der Gefässmissbildung begründet ist, sei es, dass diese gewachsen ist und nun erst störend wird.

Nach dem pathologisch-anatomischen Befund scheidet die Krankheit ans der Reihe der gewöhnlichen genuinen Epilepsien, aus. Man wird hier ohne weiteres von Hypoplasie des Gehirns bei Naevus vasculosus sprechen. Indessen sind auch wiederum Verknüpfungspunkte mit der genuinen Epilepsie vorhanden; denn in zwei der Fälle, wo die Störung 
so umfänglich ist, dass auch der ganze Schläfenlappen davon betroffen ist, findet sich Sklerose des Ammonhorns dieser Seite. Dies stützt auch meine Annahme, dass diese eine Hypoplasie und keine Gliose ist.

Was schliesslich die epileptischen Anfälle der mehr oder minder blödsinnigen, aber zum Teil gutgearteten Kranken angeht, so haben sie bei jedem meiner Kranken etwas Eigenartiges. Sie sind meist einseitige kleine Anfälle, können sich aber auch verallgemeinern.

Das Genauere ergeben die Krankengeschichten, die ich nunmehr als Anhang folgen lasse.

A. H., geb. den 6. 3. 1866, Maurerstochter, evang., ledig, stammt von Eltern, die Geschwisterhinder sind. Ihre Geschwister sind gesund. Der erste Anfall trat im 7. Lebensmonat ein (kurz vor Durchbruch des ersten Sehneidezahns). Die Krämpfe sind anfänglich alle Monate aufgetreten, einmal blieben sie $1 \frac{1 / 2}{2}$ Jahre fort. In den ersten 2 bis 3 Jaluren ging dem Ausbruch jedes Anfalles ein heftiger Schrei voraus. Pat. selbst merkt das Herannahen dés Anfalles nie, sie hat sich dabei manchmal leicht verletat, auch in die Zunge gebissen und Urin unter sich gehen lasser. Im Anschluss an die Anfälle pflegte sich eine stunden- bis tagelange Benommenheit und Schlafsucht einzustellen. Neben den Krämpfen kamen Schwindelanfälle vor. Pat. hat infolge der Anfälle mehrmals wieder das Laufen verlernt. Im 3. Lebensjahre hat sie ein hitziges Nervenfieber durchgemacht, es bildete sich innerhalb kurzer Zeit eine davernde lähmungsartige Schwäche in der linken Hand und dem linken Fusse aus, so dass sie Handarbeiten nicht erlernen konnte und den Fuss nachzieht. Im 2. Lebensjahre lernte die Kranke sprechen. In der Schule hat sie nur schreiben gelernt. Lesen und Rechnen war ihr nicht beizubringen. Thr Gedächtnis erschien gut, in ihren Anschauungen blieb sie immer naiv und schwachsinnig. Ueber die Tageszeit wusste sie nie recht Bescheid, sie war ruhig und guter Gemütsart.

Ihre Aufnahme in die Irrenanstalt 1877 erfolgte nach schweren häufigen Krämpfen. Auch in den ferneren Jahren war sie häufig von Krampf- und Schwindelanfällen heimgesucht. Letztere bestanden in Schreckbewegungen, stierem Blick und Abwesenheit. Kurz vor Ausbruch und nach Beendigung der Krampfanfälle kramte sie eine Zeitlang zwecklos an Bettzeug herum.

Körperlicher Befund (13.12.93): Rechts im Gesicht ein grosser Naevus vasculosus. Linksseitige Parese. Kontraktur im Ellenbogen und Handgelenk, geringere Entwicklung der linken Gliedmassen:

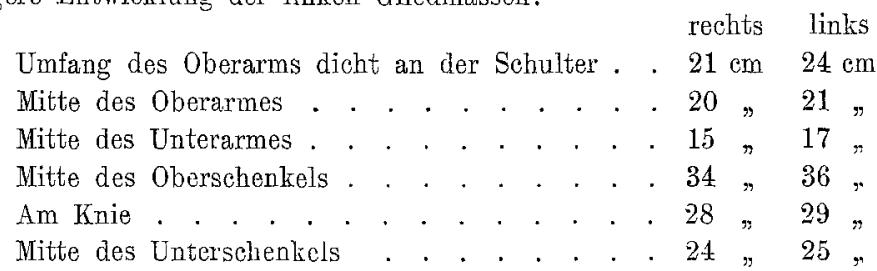

Sie tritt links schwächer auf und mit den Zehenspitzen; öfter wird TaumeIn nach der linken Seite benbachtet. Patellarreflexe gut. Fazialis rechts weniger gut 
innerviert. Linke Nasolabialfalte tiefer. Die Zunge wejcht deutlich nach links ab. Die Luft wird mit der linken. Mundhälfte ausgeblasen. Schädelumfang $51,5 \mathrm{~cm}$.

Die Kranke hielt sich viel für sich, sass untätig da, unterhielt sich mit Niemand; zuweilen war sie erregt, sehimpte dann und war bedrohlich und unzugänglich. Meist reichte sie die Hand, erwiderte den Gruss, beantwortete Fragen mit Kopfnicken oder lajggezogenem, lautem. $\mathrm{Ja}$; ab und zu brachte sie in abgebrochenen, unverständlichen Sätzen anscheinend Beschwerden gegen andere Kranke vor, wenigstens zeigte sie dabei auf die Mitkranken. An Anfällen sowohl schweren wie leichten litt sie sehr häufig und war regelmässig dabei nass. Mit Bromkalium wurde nur zeitweilig eine Verminderung der Anfälle ereicht.

Im Juli 1896 stellte sich im Anschluss an eine Lymphadenitis mit Fieber Dekubitus ein, der aller Behandlung spottete, auch nachdem das Fieber wieder geschwunden war. Sie kam dazu körperlich zurück, da sie nur wlüssigkeiten schluckte. Es ham dann ein Zustand, in dem sich an allen Stellen, wo sie adlag, Blasen bildeten; damit war verbunden Fieber:

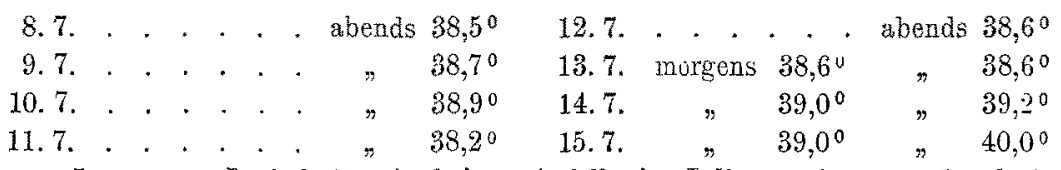

Im ganzen Juni hatte sie keinen Anfall, im Juli nur einen am 5., doch war sie oft nass. Seit dem 14. hustete sie; den 15. abends 101/2 Uhr starb sie.

Obduktion: Schädel klein, schmal, länglich, die ganze rechte Hälfte schwächer entwickelt. Nähte durchweg erhalten mit Ausnahrae der hinteren Hälfte der Sagittalnaht. Ueberall Diploe. Schädeldach schwer, maittlerer Dicke, innere Tafel rechterseits verdickt. Im Sinus longitudinalis dunkles geronnenes Blut und Speckhaut. Dura der Konvexität rechterseits innen mit einem gefässreichen Häutohen bedeckt, linkerseits innen glatt. Die Dura der Basis zeigt rechts die gleiche Veränderung wie oben. Die mittlere Schädelgrube ist rechts kleiner, die linke hintere Schädelgrube kleiner. Gehirngewicht $900 \mathrm{~g}$, Die Basilaris ist an der Vereinigungsstelle verdicht. Die grossen Nervenstämme ohne Besonderheit. Pia der Basis an den Sylvischen Gruben verdickt. Die linke Kleinbirnhälfte etwa halb so gross wie die rechte. Der rechte Schläfelappen beträchtich kleiner als der linke. Die Pia der Konrexitat linkerseits zart, von mittlerer Blutfille, glatt abziehbar, Windungen glatt, granweiss. Die Pia der rechten Seite verdiekt, die Gefässe in der Wand verdickt, geschlängelt. Die Pia sitzt in den binteren Teilen fest, vorn lässt sie sich abziehen. Die Windungen des rechten Stirnhirns sind etwas schmäler wie die des linken. Ausgesprochen ist die Mikrogyrie in den rechten Zentralwindungen. Hart fuihlt sich die vordere Zentralwindung rechts in ibrer unteren Hallte an, hart die ganze hintere Zentralwindung und die dahinter liegenden Windungen. An der Spitze des Hinterhauptlappens eine mit Kalk durchsetzte Stelle. Rechter Seitenventrikel kleiner als der linke, die Wandung glatt und getruibt, vielleicht verdickt. Der rechte Thalamus optieus etwa halb so gross, wie der linke. Sklerose des rechten Cornu Ammon. Am Rückenmark erscheint die linke Hälfte im Halsteil etwas weniger entwickelt als die rechte. 
Das Herz ist klein, Gewicht $150 \mathrm{~g}$. Lmfang der Pulmonalis 6,75, der Aorta. $5,5 \mathrm{~cm}$, die Aorta im Anfangsteil wejsslich restreift, Aortenklappen trübe, Klappen sonst zart. Die Lungen sind mit der Pleura durch einzelne Stränge verwachsen.

Umfang des linken kontrakturierten Armes beträchtlich, des linken Beines wenig geringer als die Glieder der anderen Seite.

0. S., geh. den 15. 11. 1881, Manrerssohn, ist erblich angeblich nicht belastet, erkranlste nach Angabe der Mutter im 3. Lebensjahre an einem Ausschlag auf Brust und Gesicht, wovon die Röte im Gesicht hommen soll. Im 5. Lebensjahre wurde er aus der Warteschule ohne Besinnung nach Hause gebracht. Er hatte Krämpfe, der rechte Arm und das rechte Bein waren vollständig gelähmt, das rechte Bein etwas weniger. Die Sprache war ganz verloren, während er vorher ganz gut gesprochen hatte. Bei den Anfällen fiel er plötzlich auf das Gesicht, um. dann gleich wieder aufzustehen. Solcher Anfälle kamen oft zwanzig an einem Tage. Daneben kamen aber auch Anfälle mit Zuckungen in den Gliedern vor. Durch das Fallen schwollen die Lippen an, gingen nachher immer wieder etwas zurück.

Er erwies sich in der Anstalt, in die er im Jahre $1888 \mathrm{kam}$, als ein tiefstehender, durch die Anfälle sehr mitgenommener Knabe, dessen Stimmung meist heiter und der freundlich und fügsam war. Er hatte einen ausgedehnten Naevus vasculosus der linken. Gesichtshälfte und Mundschleimhaut, die rechten Gliedmassen waren schwächer, die Sprache mangelhaft. Die linke Pupille war etwas weiter wie die rechte, die Gestalt beider nicht kreisrund. Die Reaktion beider Irides war gut. Die Patellarreflexe leicht auslösbar, lebhafter Cremasterund Bauchdeckenreflex. Das Lagegefühl, die Schmerzempfindlichkeit schien überall in Ordnung zu sein. Bei Nadelstichen im Gesicht wurden die Muskeln, besonders der rechten Gesichtshälfte, wie im Krampf zusammengezogen, wobei leichte fibrilläre Zuckungen auftraten. Er zählte sicher nur bis 15, von 10 bis 1 zu zählen, war ihm unmöglich, er las nur einzelne Buchstaben. Er war reinlich.

Im Oktober 1896 klagte er viel über Kopfschmerzen in der linken Stirnhälfte, hatte auch mehrmals Erbrechen, der Puls war schwach, 84. Dabei hatte er Fieber, das aber bald wieder schwand.

Am 25. 6. 97 wurde bemerkt, dass die Lähmung der rechten Seite zugenommen hatte. Er konnte nur mit Unterstützung gehen, schleppte das rechte Bein nach und konnte den rechten Arm so gut wie garnicht erheben. Die rechte Gesichtshälfte war schlechter innerviert. Die Zunge wurde stark zitternd nach rechts herausgestreckt. Puls kräftig, 90. Die Pupillen waren gleich weit.

Mitte August 1897 war die Lähmung des rechten Beines nur noch ganz gering, die des rechten Armes gebessert, Mitte November aber wieder sehr auffällig.

Im August 1904 trat ein Ulkus der linken Hornhaut auf und daran schloss sich eine Iridocyclitis. Das Ulkus breitete sich trotz alier Behandlung über die ganze Kornea aus.

Anfangs September 1904 erkrankte er unter Fieber mit den Zeichen der Lungenentzündung und Herzschwäche. Gleichzeitig traten gehäufte Anfälle auf und er verschied am 4. 9. 04, morgens 5 Uhr 30 Min.

Er hatte die ganzen Jahre fast Tag für Tag mit geringen Unterbrechungen einen oder mehrere Anfälle, zeitweise auch nachts. Die meisten waren leichte 
"Rucke“. Es batte aber auch ausgesprochene mit Zuckungen. Weil er dabei die Neigung hatte, auf das Gesicht zu fallen, trug er seit vielen Jahren eine gepolsterte Schutzkappe. Während der letzten Krankheit hatte er innerhalb 6 Tagen 170 Anfälle, in der Nacht vor dem Tode war er anfallsfrei.

Die 0 bduktion ergab folgendes: Naevus vasculosus faciei praec. part. sin. Haemangioma teleangiectodes piae matris sin. Hypoplasia hemisphaer. sin. Atrophia nervi opt. sin. Panophthalmia sin. Pneumonie. Pleuritis adh. Gastritis chron. Schädeldach dick, Diploe vorhanden. Rechtes Scheitelwandbein abgeflacht. Dura, längs des Sin. long. mit dem Sehädeldach verwachsen, seheint bläulich durch, ist innen glatt und glänzend. Schädelbasis in der ganzen linken Hälfte flacher als rechts. Impressiones dig. sin. weniger ausgeprägt. Die linke Schläfengrube merklich kleiner als die rechte, zeigt tief eingeschnittene Gefässfurchen. Der Boden der mittleren Schädelgrube ist stark vorgewölbt. Ebenso ist das linke Felsenbein wenig ausdrucksvoll geformt, gleich einer klumpigen Masse. Gehirngewicht: $1110 \mathrm{~g}$.

Die linke Hirnhälfte ist deutlich kleiner als die rechte. Die Pia ist links überall von starken Gefässstämmen durchzogen und durch übermässige Gefässbildung dunkelrot gefärbt und verdickt. Sonst ist die Pia feucht und glatt abziehbar. Die Gefässe der Basis sind eng, regelmässig verzweigt. Das Corpus mam. sin. ist flacher als das rechte. Das linke Cornu Ammon. schmal und verhärtet.

Der linke Optikus ist grau und schmal. Die zentralen Ganglien der linken Seite sind kleiner als die der rechten. Durchschnitte durch Pons, Medulla obl. und Riückenmark ohne Besonderheiten.

Schädelmasse: $14 \times 17 \times 51$. Thymus in Resten vorhanden. Umfang der Aorta 5,5, der Pulmonalis $6 \mathrm{~cm}$. Milz $9 \times 5 \times 2,5 \mathrm{~cm}$.

F. H., geb. am 29. 3. 1871, Kaufmannstochter, mit einem grossen Naevus pigmentosus, der fast das ganze Gesicht, die Rückseite des Thorax und die rechte Vorderseite der Brust einnimint, hatte im 3. Lebensjahre, angeblich nachdem sie sich den Kopf an der Bettstelle gestossen hatte, eine Gehirnentzündung und war nach wochenlangem Krankenlager anscheinend wieder völlig gesund, als nach einigen Wochen Zuckungen in den Händen und Armen, nach Jahresfrist richtige Krampfanfälle eintraten und von da $a b$ völlige Verblödung sich entwickelte. Die Anfälle häuften sich mit den Jahren, waren mit Bewusstlosigkeit und Einnässen verbunden. Sio musste stets gefüttert werden.

Aufgenommen in Wuhlgarten am 28. 9. 1898.

Schädel klein, Gesichtszüge grob. Die linke Gesichtshälfte stärker entwickelt wie die rechte. Pupillen mittelweit, gleich, Reaktion gut. Gaumen hoch und schmal. Die Zähne fehlen fast vollständig. Die Zunge ist an den Rändern mit Bissnarben versehen. Am Thorax ist die linke Hälfte stärker entwickelt. Herztöne rein. Rechter und linker Arm gelähmt. Der rechte wird an den Brustkorb angezogen gehalten, ist im Ellenbogen- und Handgelenk gebeugt, die Finger sind in die Hohlband eingeschlagen, alles in Kontraktur. Der linke Arm ist im Schultergelenk beweglich, im Ellenbogengelenk gestreckt gehalten und steif, die Hand befindet sich in Ueberstreckung. Das Gefühl seheint unversehrt zu sein. Auf Kneifen antwortet sie mit Schreien. Die Beine sind ge- 
Mähmt, befinden sich in allen Gelenken in starrer Beugestellung. Die Muskulatur ver Glieder ist atrophisch. Das Gefuhl ist anseheinend erhalten, ebenso die Patellarreflexe. Es besteht Blasen- und Mastdarmlähmung. Die Kranke ist vollständige ldiotin, sie sieht und bürt, versteht nichts, kennt die Ungelpung nicht. Sie hat fast Tay für Tag oft melirere Anfälle, auch manchmal sogenannte Schwindelanfä̈le. Die Anfälle verlaufen mit Zuckungen in allen Gliedern, Bewusstlosigkeit und Einnässen.

Ausgangs Dezember 1398 stellte sich Dekubitus ein, der bald wieder heilte. Am 15. 2. 1899 erkrankte sie unter Fieber an Durchfall und Lungenerscheimungen und starb am 18 . abend $101 / 2$ Uhr.

Obduktionsbefund. Kopfmasse: Umfang 48, grösste länge 16, grösste Breite $13,5 \mathrm{~cm}$. Gehirngewicht $1032 \mathrm{~g}$. Schädeldach im rechten schrägen Durchmesser, hauptsächlichlich durch eine Verkleinerung der rechten Hälfte des Stirnbeins verkürat. Nähte orhalten, Sebädeldach leicht, über mitteldick, die rechte Hälite des Stimbeins doppelt so dick wie die linke. Viel Diploe. Diese fehlt längs der Koronarnaht, längs der Mittellinie des Stirnbeins, in der hinteren Hälfte des linken Seitenwandbeins, der linken Hälfte der Occipitalschnppe. Die Gefässfurchen sind scharf abgesetzt. Der Schädel ist hoch gewölbt. Die Dura ist am rechten Stirnteil verdickt, innen glatt und glänzend. Die Dura der Basis ebenso, der Klivus ist steil, im Sinus geronnenes Blut und Speckhaut. Die rechte mittlere Schädelgrube ist stärker wie die linke. Das linke Felsenbein bildet mit der Mittellinie einen spitzeren Winkel als das rechte. Das orbitale Gewölbe ist rechts bedeutend höber, die hintere rechte Grosshimhälfte ist in allen Pichtungen kleiner wie die linke, sie bleibt vorn, hinten und seitlich um ungefähr $1,5 \mathrm{~cm}$ zurüek. Das rechte Kleinhirn ist ebenfalls stark verringert. Die Pia der Basis ist in der Mitte getrïbt und verdickt. Die Gefässe regelrecht angeordnet, enthalten verschiebliches Blut. Das rechte Corpus mamillare erseheint etwas flacher wie das linke. Der rechte Tractus opticus ist halb so breit wie der linke. Die Pia dex Konvexität der linken Memisphäre ist zart, die der rechten an der mittleren Kante auffallend verdickt und getrübt. Die Seitenventrikel sind von mittlerer Weite, der rechte etwas enger wic der linke. Der Plexus chorioideus der rechten Seite fühlt sich an einer Stelle hart an, scheint bindegewebig entartet zu sein. Thein aufallender Untersehied in den Hälften cles Pons und der Medulla. Die reohte Kleinhirnhälfte ist gleichmässig in der Masse, auch in den Kernen verkleinert. Das Ependym der Seitenventrikel ist weisslich getrïbt, Thalamus opticus und Corpus striatum dextrum verkleinert, die Ammonshörner gleich entwickelt. Der rechte Schläfenlappen ist im ganzen erweicht, die weisse Substanz nahezu geschwunden, ebenso verhält sich der rechte Hinterhauptslappen, in beiden finden sich viele Kallkkörnchen. Die weisse Substanz ist sonst gut entwichelt, rechts etwas geringer. Die Rinde ist nicht auffällig verschmälert, aulsgenommen am rechten Schläfen- und Hinterhauptslappen, denen sich noch die Spitze des Stirnlappens anschliesst, woselbst ebenfalls viel Kalkkörnehen vorhanden sind. Am Rückenmark ist nichts Besonderes. Von der weiteren Seltion ist Abstand genommen worden. 